\title{
Rapid infusion of esketamine for unipolar and bipolar depression: a retrospective chart review
}

\author{
This article was published in the following Dove Press journal: \\ Neuropsychiatric Disease and Treatment \\ 21 June 2017 \\ Number of times this article has been viewed
}

\section{Fernanda S Correia-Melo' \\ Felipe C Argolo' \\ Lucas Araújo-de-Freitas ${ }^{1,2}$ \\ Gustavo Carneiro Leal' \\ Flávio Kapczinski ${ }^{3}$ \\ Acioly Luiz Lacerda ${ }^{4}$ \\ Lucas C Quarantini',2}

'Psychiatry Service, University Hospital, Federal University of Bahia, Salvador, Brazil; ${ }^{2}$ Postgraduate Program in Medicine and Health, Federal University of Bahia, Salvador, Brazil; ${ }^{3}$ Department of Psychiatry, Federal University of Rio Grande do Sul, Porto Alegre, Brazil; ${ }^{4}$ Department of Psychiatry, Federal University of São Paulo, São Paulo, Brazil
Correspondence: Lucas C Quarantini Hospital Universitário Professor Edgard Santos, Serviço de Psiquiatria, 3 andar, 40I I 0-060 Salvador, BA, Brazil Fax +55 7I $35064 \mid 44$ Email lcq@ufba.br
Background: This study evaluated efficacy and safety of intravenous subanesthetic doses of esketamine using an administration time of 10 minutes in patients with treatment-resistant depression and bipolar depression.

Methods: A retrospective chart review was conducted to identify patients who met the inclusion criteria for treatment-resistant depression and bipolar depression according to Diagnostic and Statistical Manual of Mental Disorders, Fourth Edition, Text Revision criteria, and these patients received rapid infusion of esketamine between June 2012 and December 2015. The Montgomery-Åsberg Depression Rating Scale (MADRS) was administered to measure and score depressive symptom severity before infusion and at 24 hours, 72 hours, and 7 days after infusion. In addition, Clinical Global Impression scale was administered before and 7 days after esketamine infusion.

Results: Esketamine was administered to 30 patients. A total of 27 patients met the inclusion criteria and had MADRS evaluation data, which showed that 23 had unipolar and 4 had bipolar depression. Thirteen patients (48.1\%) showed therapeutic response (MADRS reduction $\geq 50 \%$ ) within 1 week ( 7 days) of intervention. Remission (MADRS $<7$ ) was observed in 10 patients $(37.0 \%)$ in the same period. Therapeutic response and remission frequencies were seen in $16(59.3 \%)$ and $11(40.7 \%)$ patients, respectively, within 24 hours following drug infusion. The most relevant side effect observed during the esketamine infusion was dissociative symptoms ranging from mild to severe, which was reported by $11.1 \%$ of patients as a very disturbing experience.

Limitations: This study was done retrospectively, had a small sample size, and there was no comparative group.

Conclusion: The present study demonstrates that rapid infusion of esketamine is possibly not the optimal choice to administer this drug for treatment-resistant depression due to tolerability reasons. Further controlled studies are required to investigate efficacy, safety, and tolerability profiles among the different types of ketamines and methods of using this drug in depressed patients.

Keywords: tolerability, $S$-(+)-ketamine, treatment-resistant depression, dissociative symptoms, efficacy, safety

\section{Introduction}

Major depressive disorder is a debilitating mental illness that affects millions of subjects worldwide, leading to severe clinical and socioeconomic consequences. ${ }^{1}$ About onethird of patients with major depressive disorder do not achieve remission from currently available treatments and could be considered as having treatment-resistant depression (TRD), which is associated with chronicity, higher morbidity, functional disability, and economic loss. ${ }^{2-6}$ In addition, TRD patients are affected by more comorbidities than non-TRD patients. ${ }^{7}$ 
Current limitations of available antidepressants include delays in therapeutic effects from several weeks to months and low remission rates. ${ }^{8,9}$ Revolutionizing the therapeutic options for TRD, ketamine (or $R S$-( \pm )-ketamine), an N-methyld-aspartate (NMDA) receptor antagonist, administered at subanesthetic doses can elicit robust, rapid, and sustained antidepressant effects in TRD. ${ }^{10}$ The majority of clinical trials that describe rapid antidepressant effects on TRD involve the $R S$ - $( \pm)$-ketamine. ${ }^{11-15}$ A recently published trial performed by Singh et $a{ }^{17}$ reported the rapid antidepressant action of esketamine $(S$ - $(+)$-ketamine) after a 40 -minute intravenous (IV) infusion of either $0.20 \mathrm{mg} / \mathrm{kg}$ or $0.40 \mathrm{mg} /$ $\mathrm{kg}$ dose in patients with TRD. It was also demonstrated that a lower dose produced a better tolerability while maintaining efficacy.

Recently, esketamine was referred to as a less dissociative drug than $R S$-( \pm -ketamine. ${ }^{12}$ However, this comparison has been insufficiently studied since there are only a few publications regarding esketamine use on TRD and with limited diversity of posology. ${ }^{16,17}$ Consequently, there is little evidence about the safety and tolerability profile of esketamine and its dissociative properties when used in TRD patients.

A crucial point related to ketamine use is safety, especially due to cardiovascular risks and abusive misuse. ${ }^{10}$ Therefore, its administration requires clinical monitoring and other supporting services under medical supervision. ${ }^{10,13,14}$

Most protocols for ketamine administration adopt a timeframe of 40 minutes for infusion. However, this parameter is not supported by evidence, considering that in anesthesiology it is common to use a higher dose of $R S$ - $( \pm)$-ketamine $(>2 \mathrm{mg} / \mathrm{kg}$ ) with faster speed of infusion lasting 5-10 minutes for IV administration, and this is particularly true regarding esketamine. ${ }^{18,19}$ We examined the efficacy, safety, and tolerability of off-label IV use of esketamine in TRD.

\section{Materials and methods}

This retrospective chart review was carried out at the inpatient unit at the Psychiatry Service at the University Hospital - Universidade Federal da Bahia, Salvador, Brazil. Patients were assessed between June 2012 and December 2015, with the diagnosis of MDD and dipolar disorder being based upon Diagnostic and Statistical Manual of Mental Disorders, Fourth Edition, Text Revision criteria (Diagnostic and Statistical Manual of Mental Disorders, Fourth Edition, Text Revision [DSM-IV-TR], 2000); Montgomery-Åsberg Depression Rating Scale (MADRS) ${ }^{20}$ was administered to measure depressive symptom severity before infusion and at 24 hours, 72 hours, and 7 days after infusion of ketamine, and the Clinical Global Impression scale (CGI) was administere ${ }^{21}$ before and at 7 days after infusion.

TRD was defined as failure to respond to 2 or more trials using antidepressant therapy in adequate doses, with durations of at least 6 weeks each.

All the participants were undergoing naturalistic treatment for depression and comorbidities, according to clinical demand. The clinical protocol excluded the use of esketamine in patients if they met any of the following criteria: psychotic disorder, previous dissociative disorders, comorbid dementia, substance use disorders, and/or uncontrolled hypertension.

The primary endpoints were remission of depressive symptoms and therapeutic response, defined as at least $50 \%$ improvement from baseline depression score. All adverse effects that emerged during infusion and at subsequent observation periods were also registered.

All subjects were treated with esketamine (Ketamin ${ }^{\circledR}$ Dextrocetamine hydrochloride Injectable Solution $50 \mathrm{mg} / \mathrm{mL}$. Cristalia Produtos Químicos Farmarcêuticos Ltda, Itapira, Brazil) at a standard dose of $0.25 \mathrm{mg} / \mathrm{kg}$ administered IV for a duration of 10 minutes.

The study was approved by the local Institutional Review Board (Complex University Hospital Professor Edgard Santos - Federal University of Bahia - Number: 43645915.1.10000.0049). The Research Ethics CommitteeUniversity Hospital Prof Edgard Santos (Local Institutional Review Board) waived the need for patient consent for this retrospective review since all data were anonymized and kept confidential.

\section{Statistical analysis}

A summary of statistical parameters (mean, standard deviation, median, interquartile range, minimum, and maximum) are reported for the MADRS score at each point in time. Quantitative temporal differences among time points were tested, controlling for clinical comorbidities, through a linear mixed effects model. The model was fit with one fixed intercept, Time and Presence of Clinical Comorbidity as fixed effect, and one random intercept for every patient, allowing for the analysis of data without data imputation or patient exclusion from the sample. ${ }^{22}$ Assumptions of normality of the residuals and homogeneity of variance were verified through QQ Plot and graphical analysis of predicted values and residuals. Mean differences were estimated from the model through Tukey contrasts.

Absolute and relative (percentage) frequencies of remission of depressive symptoms and therapeutic response are reported. All relative frequencies (percentage) are related to 
the initial number of patients included. MADRS scores among these groups are also reported. All analyses were conducted in R Environment and Programming Language (version 3.3.1); mixed effects model was fit using lme4 (version 1.1.12) package with restricted maximum likelihood method (REML).

\section{Results}

\section{General characteristics}

Sociodemographic characteristics are displayed in Table 1.

\section{Depressive symptoms and baseline characteristics}

Initially, our sample consisted of 30 patients, but MADRS data was not available for 3 of them, hence 27 were

Table I General clinical and epidemiological characteristics of the patient sample undergoing treatment with rapid infusion of esketamine

\begin{tabular}{|c|c|}
\hline Variables & Mean (min-max) \\
\hline Age & $51.0(42.0-64.0)$ \\
\hline \multirow[t]{2}{*}{ Years of education } & $15.0(15.0-16.0)$ \\
\hline & $\mathbf{N}(\%)$ \\
\hline \multicolumn{2}{|l|}{ Civil status } \\
\hline Single & $7(25.0)$ \\
\hline Married & $14(50.0)$ \\
\hline Divorced & $3(10.7)$ \\
\hline Widow & $2(7.1)$ \\
\hline Male sex & $17(60.7)$ \\
\hline \multicolumn{2}{|l|}{ Ethnicity (self-declared) } \\
\hline Caucasian & $13(46.4)$ \\
\hline Afro-Brazilian & $13(46.4)$ \\
\hline Nondeclared & $2(7.2)$ \\
\hline \multicolumn{2}{|l|}{ Occupation } \\
\hline Student & I (3.6) \\
\hline Remunerated occupation & $15(53.6)$ \\
\hline Nonremunerated occupation & $12(42.8)$ \\
\hline \multicolumn{2}{|l|}{ Main diagnosis } \\
\hline Depression unipolar & $22(78.6)$ \\
\hline Depression bipolar & $6(21.4)$ \\
\hline \multicolumn{2}{|l|}{ Psychiatric comorbidities } \\
\hline OCD & $4(14.3)$ \\
\hline Social phobia & $4(14.3)$ \\
\hline PTSD & $2(7.1)$ \\
\hline GAD & $2(7.1)$ \\
\hline Panic disorder & $2(7.1)$ \\
\hline ADHD & $2(7.1)$ \\
\hline Agoraphobia & I (3.6) \\
\hline Benzodiazepine use & $13(46.4)$ \\
\hline \multicolumn{2}{|l|}{ Clinical comorbidities } \\
\hline Cardiovascular disease & $7(25.0)$ \\
\hline Endocrine disease & $5(17.9)$ \\
\hline Cancer & I (3.6) \\
\hline
\end{tabular}

Abbreviations: OCD, obsessive compulsive disorder; PTSD, posttraumatic stress disorder; GAD, generalized anxiety disorder; ADHD, attention-deficit hyperactivity disorder. finally included. Therefore a retrospective chart review is reported for 23 patients with TRD and 4 with bipolar depression, who all received a low-dose of esketamine administered very quickly: time duration 10 minutes.

The majority of participants had chronic depressive symptoms, and all of them had presented at least 2 failed trials using antidepressants.

The patients presented a high MADRS mean score (36.2 \pm 7.6$)$ prior to intervention. The median of onset age of major depressive episode was 38 years, with half of the patients ranging from 26.0 to 50.0 years. All patients presented a lifetime history of major depressive episode with an average of 4.0 episodes (interquartile range: 2.8-6.0). Four patients $(14.8 \%)$ were bipolar. The most common psychiatric comorbidities were obsessive compulsive disorder (11.1\%) and social phobia (11.1\%). All patients from this study were treated with antidepressants, 20 (74.1\%) used atypical antipsychotics, and only 5 (18.5\%) used lithium (the others had prior therapeutic failure with this drug and/or had contraindications to using it). Benzodiazepine usage was present in 13 patients (48.1\%). Most subjects (17 individuals; 63.0\% of the sample) did not have medical comorbidities. Among those who presented medical comorbidities, cardiovascular diseases were the most common (33.3\%), followed by endocrine diseases $(18.5 \%)$ and cancer $(7.4 \%)$.

\section{Therapeutic response}

Average values of MADRS showed reductions during follow-up time. A summary of the statistics is displayed in Table 2 and Figure 1.

Thirteen patients (48.1\%) displayed therapeutic response (MADRS score reduction $\geq 50 \%$ ) within 1 week ( 7 days) after intervention. Remission (MADRS $<7$ ) was observed in 10 patients $(37.0 \%)$ in the same time period. Therapeutic response and remission frequencies were observed in 16 patients $(59.3 \%)$ and 11 patients $(40.7 \%)$, respectively, within 24 hours after drug infusion.

The mixed effects model converged to a unique solution (REML criterion $=716.04$; random effect $\mathrm{SD}=10.636$; fixed intercept $=34.473$ ). Fixed-effect estimate for the presence of medical comorbidity was positive (estimate $=3.397$; standard error $=4.929$ ), although not significant (Type II Wald $\left.\chi^{2}=0.475 ; d f=1 ; P=0.491\right)$. Time factor was significant (Type II Wald $\chi^{2}=66.846 ; d f=3 ; P<0.001$ ), with large estimated mean differences between the time points Before Intervention and the remaining follow-up points (24 hours BI: $-18.73 \pm 2.31 ; 72$ hours $-\mathrm{BI}:-17.51 \pm 2.34 ; 7$ days BI: $-17.16 \pm 2.34$; all $P$-values $<0.001$ ). 


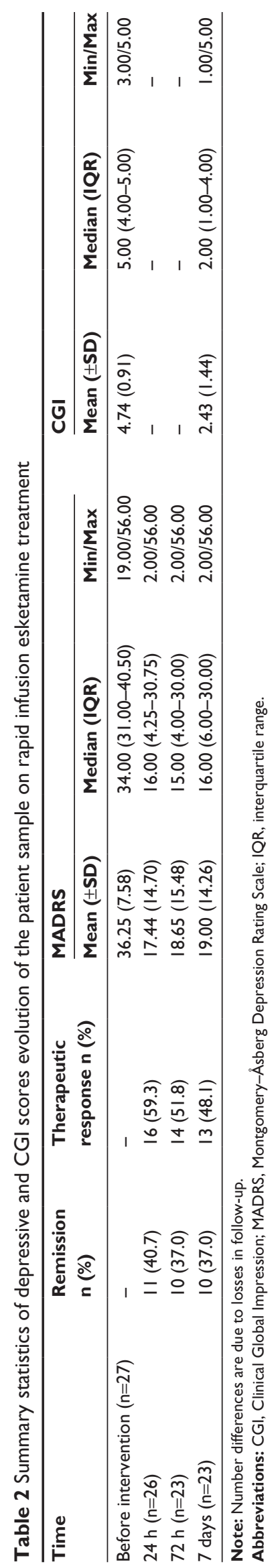

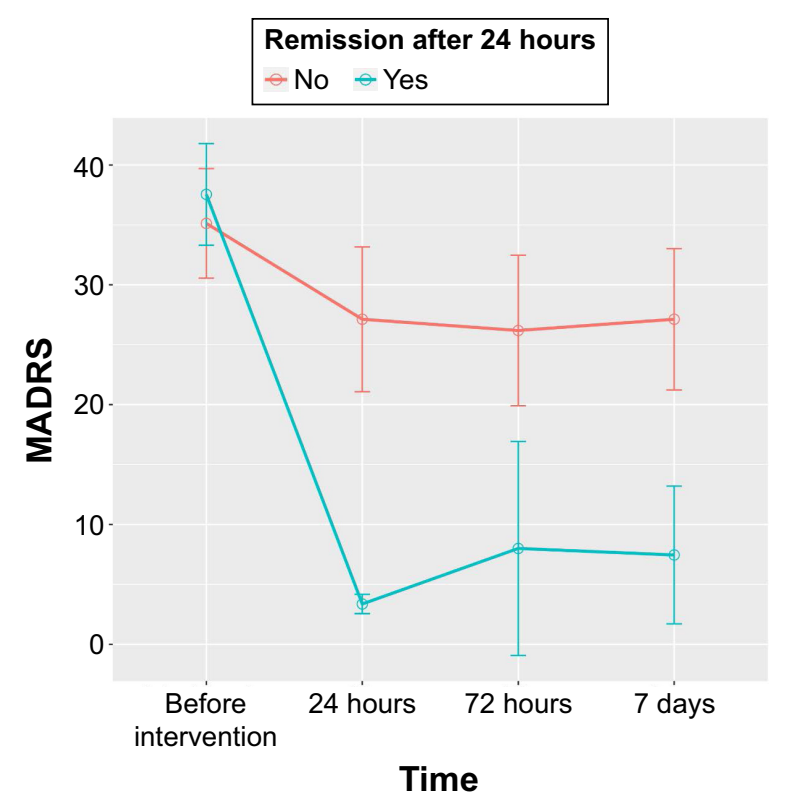

Figure I Temporal evolution of therapeutic remission of the patient sample upon rapid infusion esketamine treatment.

Note: Points represent means, and error bars show $95 \%$ confidence intervals estimated from standard errors.

Abbreviation: MADRS, Montgomery-Åsberg Depression Rating Scale.

\section{Safety}

Vital signs, electrocardiograms, and clinical laboratory assessments were within normal ranges. No deaths occurred in the cases reviewed. No clinically significant changes in laboratory tests, electrocardiograms, or physical examinations were observed in the week following esketamine infusion.

The most relevant side effect observed was mild to severe dissociative symptoms. Three patients (11.1\%) had psychomimetic effects during esketamine infusion, which presented as severe dissociative symptoms, and two of these cases showed remission within 3 weeks after esketamine administration.

\section{Discussion}

Previous clinical trials described the rapid action of NMDA antagonist ketamine after a single infusion in patients with TRD $^{11,15,23,24}$ as well as the variable duration of antidepressant effects ranging from 1 to several weeks. ${ }^{15,25}$ Although it represents a major advance in the treatment of TRD, many questions remain about the efficacy and tolerability of this drug in depressed patients.

To our knowledge, this is the first report regarding rapid infusion time (10 minutes) of esketamine in TRD patients. The present results suggest that, despite the robust efficacy obtained in these naturalistically treated patients, this rate of drug administration produced certain severe dissociative 
side effects. These results were not modified by the presence of medical and psychiatric comorbidities. The finding that esketamine use may be associated with highly distressing symptoms related to the dissociative experience challenges previous studies that characterize the esketamine as more tolerable than the $R S-( \pm)$-ketamine. ${ }^{26}$ In four patients, the severity of side effects seemed to contradict the improvement of depressive symptoms.

The presence of dissociative symptoms has been observed as a predictor for a better antidepressant effect of ketamine. However, this is a limited finding from only one study with many limitations such as secondary data analysis, combined diagnostic groups, potential unblinding, and small sample sizes. ${ }^{27}$

The present findings should be interpreted with caution since several limitations inherent to using a retrospective design are present. They include the lack of a control group and lack of randomization. As this was not a controlled study and the subjects had taken several other medications that might modify antidepressant effects and pharmacokinetics of esketamine, we cannot assure whether the efficacy is related to esketamine use alone or is a result of combined effects of esketamine and concomitant medications. Additionally, no standardized clinical measures were used to evaluate psychomimetic adverse effects. Nevertheless, due to these limitations, a prospective, controlled study using different doses and time of infusion is strongly required to confirm the efficacy of the esketamine as a feasible option for eliciting fast-acting antidepressant effects, with a reasonable side effect profile.

\section{Conclusion}

The present case series suggests that rapid infusion of esketamine has similar efficacy when compared with $R S$ - $( \pm)$-ketamine data in the literature. On the other hand, esketamine seems to have poor tolerability when compared to the racemic formulation. However, the infusion rate used in the present study may explain, at least in part, this poor tolerability. Randomized, controlled studies comparing efficacy and tolerability of $S$-(+)- and $R S$-( $( \pm)$-ketamine are needed to confirm the present findings.

\section{Disclosure}

The authors report no conflicts of interest in this work.

\section{References}

1. Kessler RC, Berglund P, Demler O, et al. The epidemiology of major depressive disorder: results from the National Comorbidity Survey Replication (NCS-R). JAMA. 2003;289(23):3095-3105.
2. Fava M, Davidson KG. Definition and epidemiology of treatmentresistant depression. Psychiatr Clin North Am. 1996;19(2):179-200.

3. Gaynes BN, Warden D, Trivedi MH, Wisniewski SR, Fava M, Rush AJ. What did STAR*D teach us? Results from a large-scale, practical, clinical trial for patients with depression. Psychiatr Serv. 2009;60(11): 1439-1445.

4. Fervaha G, Foussias G, Takeuchi H, Agid O, Remington G. Motivational deficits in major depressive disorder: cross-sectional and longitudinal relationships with functional impairment and subjective well-being Compr Psychiatry. 2016;(66):31-38.

5. Gu CZ, He HL, Duan HF, Su ZH, Chen H, Gan JL. Predictors of neurocognitive impairment at 2 years after a first-episode major depressive disorder. Compr Psychiatry. 2016;(68):24-33.

6. Rush AJ, Trivedi MH, Wisniewski SR, et al. Acute and longer-term outcomes in depressed outpatients requiring one or several treatment steps: a STAR*D report. Am J Psychiatry. 2006;163(11):1905-1917.

7. Kubitz N, Mehra M, Potluri RC, Garg N, Cossrow N. Characterization of treatment resistant depression episodes in a cohort of patients from a US commercial claims database. PloS One. 2013;8(10):e76882

8. Frazer A, Benmansour S. Delayed pharmacological effects of antidepressants. Mol Psychiatry. 2002;7(Suppl 1):S23-S28.

9. Naughton M, Clarke G, O’Leary OF, Cryan JF, Dinan TG. A review of ketamine in affective disorders: current evidence of clinical efficacy, limitations of use and pre-clinical evidence on proposed mechanisms of action. J Affect Disord. 2014;156:24-35.

10. Galvez V, Nikolin S, Ho KA, Alonzo A, Somogyi AA, Loo CK. Increase in PAS-induced neuroplasticity after a treatment course of intranasal ketamine for depression. Report of three cases from a placebo-controlled trial. Compr Psychiatry. 2017;73:31-34.

11. Berman RM, Cappiello A, Anand A, et al. Antidepressant effects of ketamine in depressed patients. Biol Psychiatry. 2000;47(4):351-354.

12. Paul R, Schaaff N, Padberg F, Moller HJ, Frodl T. Comparison of racemic ketamine and S-ketamine in treatment-resistant major depression: report of two cases. World J Biol Psychiatry. 2009;10(3):241-244.

13. Fond G, Loundou A, Rabu C, et al. Ketamine administration in depressive disorders: a systematic review and meta-analysis. Psychopharmacology. 2014;231(18):3663-3676.

14. McGirr A, Berlim MT, Bond DJ, Fleck MP, Yatham LN, Lam RW. A systematic review and meta-analysis of randomized, double-blind, placebo-controlled trials of ketamine in the rapid treatment of major depressive episodes. Psychol Med. 2015;45(4):693-704.

15. Zarate CA Jr, Singh JB, Carlson PJ, et al. A randomized trial of an $\mathrm{N}$-methyl-D-aspartate antagonist in treatment-resistant major depression. Archiv Gen Psychiatry. 2006;63(8):856-864.

16. Pfenninger EG, Durieux ME, Himmelseher S. Cognitive impairment after small-dose ketamine isomers in comparison to equianalgesic racemic ketamine in human volunteers. Anesthesiology. 2002;96(2):357-366.

17. Singh JB, Fedgchin M, Daly E, et al. Intravenous esketamine in adult treatment-resistant depression: a double-blind, double-randomization, placebo-controlled study. Biol Psychiatry. 2016;80(6):424-431.

18. Green SM, Rothrock SG, Lynch EL, et al. Intramuscular ketamine for pediatric sedation in the emergency department: safety profile in 1,022 cases. Ann Emerg Med. 1998;31(6):688-697.

19. Sinner B, Graf BM. Ketamine. In: Urban BW SJ, Schwilden H, editors. Handbook Experimental Pharmacology. Berlin, Germany: Springer; 2008:313-333.

20. Montgomery SA, Asberg M. A new depression scale designed to be sensitive to change. Br J Psychiatry. 1979;134(4):382-389.

21. W G. Clinical Impressions Scale (CGI). In: Rush AJ, Pincus HA, First MB, et al, editors. Handbook of Psychiatric Measures. Washington, DC: American Psychiatric Association; 2000:100-102.

22. Littell RC, Pendergast J, Natarajan R. Modelling covariance structure in the analysis of repeated measures data. Stat Med. 2000;19(13): 1793-1819.

23. Diazgranados N, Ibrahim L, Brutsche NE, et al. A randomized add-on trial of an N-methyl-D-aspartate antagonist in treatment-resistant bipolar depression. Archiv Gen Psychiatry. 2010;67(8):793-802. 
24. DiazGranados N, Ibrahim LA, Brutsche NE, et al. Rapid resolution of suicidal ideation after a single infusion of an N-methyl-D-aspartate antagonist in patients with treatment-resistant major depressive disorder. J Clin Psychiatry. 2010;71(12):1605-1611.

25. Zarate C, Machado-Vieira R, Henter I, Ibrahim L, Diazgranados N, Salvadore G. Glutamatergic modulators: the future of treating mood disorders? Harvard Rev Psychiatry. 2010;18(5):293-303.
26. Mathew SJ, Shah A, Lapidus K, et al. Ketamine for treatment-resistant unipolar depression. CNS Drugs. 2012;26(3):189-204.

27. Luckenbaugh DA, Niciu MJ, Ionescu DF, et al. Do the dissociative side effects of ketamine mediate its antidepressant effects? J Affect Disorders. 2014;(159):56-61.

\section{Publish your work in this journal}

Neuropsychiatric Disease and Treatment is an international, peerreviewed journal of clinical therapeutics and pharmacology focusing on concise rapid reporting of clinical or pre-clinical studies on a range of neuropsychiatric and neurological disorders. This journal is indexed on PubMed Central, the 'PsycINFO' database and CAS, and is the official journal of The International Neuropsychiatric Association (INA). The manuscript management system is completely online and includes a very quick and fair peer-review system, which is all easy to use. Visit http://www.dovepress.com/testimonials.php to read real quotes from published authors. 